\title{
Quantitative assessment of factors affecting the sensitivity of a competitive immunomicroarray for pesticide detection
}

\author{
Erik Belleville ${ }^{1}$, Martin Dufva ${ }^{1}$, Jens Aamand ${ }^{2}$, Leif Bruun ${ }^{3}$, and Claus B.V. Christensen ${ }^{1}$
}

BioTechniques 35:1044-1051 (November 2003)

\begin{abstract}
Analytical protein microarrays offering highly parallel analysis can become an invaluable tool for a wide range of immunodiagnostic applications. Here we describe factors that influence the sensitivity of a competitive immunomicroarray that quantifies small molecules; in this case, the pesticides dichlobenil metabolite 2,6-dichlorobenzamide (BAM) and atrazine. Free pesticide concentrations in solution are quantified by the competitive binding of fluorescence-conjugated monoclonal antibodies to either surface-immobilized pesticide hapten-protein conjugates or pesticides in solution. We investigated the influence of antibody labeling techniques, microarray substrates, and spotting and incubation buffers. The results showed that microarrays immobilized on EasySpot or in-house fabricated agarose substrates printed with Genetix Amine Spotting Solution resulted in optimum results when the arrays were incubated with the sample/antibodies diluted in a Tris buffer supplemented with $0.05 \%$ each bovine serum albumin (BSA) and Tween ${ }^{\circledR} 20$. Furthermore, the application of directly labeled primary antibodies allowed for better sensitivity compared to secondary polyclonal antibody quantification.
\end{abstract}

\section{INTRODUCTION}

Developments in biological and chemical analysis involve the improvement and miniaturization of existing techniques to increase sensitivity, sample capacities, and throughput, while, at the same time, dramatically decrease assay costs, reagent consumption and, most importantly, test times. One of the first analytical breakthroughs arrived with the use of DNA chips, which can analyze a sample for thousands of different nucleic acids simultaneously (1). Protein microarrays have been developed for detecting protein-protein interactions, enzyme targets, and proteinsmall molecule interactions $(2,3)$. An advantage of downsizing immunoassays is an increased sensitivity in comparison to conventional macroscopic immunoassays $(4,5)$. Antigen microarrays have been used for the detection of circulating antibodies in clinical samples [e.g., microarrays of allergens for the detection of serum allergen-specific immunoglobulin $\mathrm{E}$ (IgE)] (6). Furthermore, a variety of protein microarray applications such as immobilized antibody arrays screening protein ligands (7), recombinant antibody arrays (8), autoimmune diagnostic ELISA arrays (9), enzymeinhibition arrays (10), as well as first protein arrays for relative quantification (11) have been reported.

For the development of an immunomicroarray for pesticide analysis, we evaluated different fluorescent labeling techniques for monoclonal antibodies (MAbs), primary and secondary signal quantifications, sample buffer compositions, spotting buffers, and several commercial and in-house fabricated microarray substrates of various surface chemistries for their effects on array performance.

\section{MATERIALS AND METHODS}

\section{Pesticides and Haptens}

The 2,6-dichlorobenzamide (BAM), atrazine, and hydroxy-atrazine (Table 1) were from Riedel-de Haën (Seelze, Germany). BAM, atrazine, and hy- droxy-atrazine haptens, as well as the anthraquinone (AQ) conjugate surface linker were synthesized by Exiqon A/S (Vedbaek, Denmark) as described by Bruun et al. $(12,13)$. Hapten-ovalbumin (OA)-AQ conjugates were produced by GEUS (Copenhagen, Denmark) as previously described. $(12,13)$.

\section{Immunomicroarray Antibodies}

The MAbs, anti-BAM, and antiatrazine were generated by Statens Serum Institut (Copenhagen, Denmark) as previously described (13). The MAbs were selected on the basis of titer, affinity, and specificity. A secondary $\mathrm{Cy}^{\mathrm{TM}}$ 3-labeled goat-antimouse polyclonal antibody (SigmaAldrich, Vallensbaek, Denmark) with a fluorochrome/antibody (F/A) labeling ratio of 5.4 was used for secondary binding studies.

\section{Fluorescent Monoclonal Antibody Labeling}

Anti-BAM and anti-atrazine MAbs

\footnotetext{
${ }^{1}$ Technical University of Denmark, Lyngby, ${ }^{2}$ Geological Survey of Denmark and Greenland, Copenhagen, and

${ }^{3}$ Statens Serum Institut, Copenhagen, Denmark
} 
Table 1. Microarray Substrates

\begin{tabular}{|c|c|c|c|c|}
\hline & Product & $\begin{array}{l}\text { Reactive Surface } \\
\text { Linker Groups }\end{array}$ & Supplier & $\begin{array}{l}\text { Price per Substrate } \\
\qquad(€ / \$)\end{array}$ \\
\hline$A$ & Agarose & unactivated: hydroxyl & Fabricated in house according to & $0.05 / 0.06$ \\
\hline $\mathrm{C}$ & CSA & amino silane & TeleChem International & $0.90 / 1.02$ \\
\hline $\mathrm{F}$ & GAPS $\|^{\circledR}$ & amino silane & Corning & $9.00 / 10.19$ \\
\hline G & Genorama SAL-1 & $\begin{array}{l}\text { amino silane and } \\
\text { phenylene-thiocyanate }\end{array}$ & Asper Biotech & $10.00 / 11.32$ \\
\hline $\mathrm{H}$ & Nexterion ${ }^{\circledR}$ & amino silane & Schott (Mainz, Germany) & 10.00/11.32 \\
\hline
\end{tabular}

were labeled with either a Cy5 monoreactive dye for proteins or a $\mathrm{Cy} 5$ maleimide monoreactive dye for MAbs (both from Amersham Biosciences, Buckinghamshire, UK) according to the manufacturer's instructions. Purification with Micro Bio-Spin P30 ${ }^{\circledR}$ Tris Chromatography columns (Bio-Rad Laboratories, Hercules, CA, USA) at $1000 \times g$ removed unreacted Cy5 labels, and individual F/A ratios were determined spectrophotometrically, according to manufacturers' instructions. Briefly, absorbance was measured at $280 \mathrm{~nm}$ for the protein (MAb) concentration and at $650 \mathrm{~nm}$ for the Cy5 fluorochrome concentration and used for the calculation of final F/A ratios.

\section{Immunomicroarray Quantification}

The pesticide immunomicroarray is derived from the microplate-based ELISA that has been previously described $(12,13)$. Using a QArray ${ }^{\circledR}$ microarray printer (Genetix, New Milton, UK), 10 arrays of 6 spots each of BAM, atrazine, and hydroxy-atrazine (negative controls) hapten conjugates were printed onto various microarray substrates (Table 1). Microarrays were printed at final conjugate concentrations of $0.5 \mathrm{mg} / \mathrm{mL}$ and diluted $1 \times$ in the different spotting buffers that were compared, including Genetix Amine Spotting Solution, $300 \mathrm{mM}$ phosphate, $150 \mathrm{mM}$ borate, $150 \mathrm{mM}$ phosphatebuffered saline (PBS) (all Sigma-Aldrich), and Milli- $\mathrm{Q}^{\circledR}$ water (Millipore, Waltham, MA, USA). Spot volumes were approximately $1 \mathrm{~nL} / \mathrm{spot}$ and delivered by a CMP3 Chipmaker pin ${ }^{\circledR}$ (TeleChem International, Sunnyvale, CA, USA). Covalent hapten conjugate surface immobilization was established through AQ-catalyzed photocoupling of the hapten-OA-AQ conjugates to the glass surface during $30 \mathrm{~s}$ of ultraviolet exposure at $254 \mathrm{~nm}$ in a Stratalinker ${ }^{\circledR}$ 2400 (Stratagene, La Jolla, CA, USA).

Following immobilization, the microarray substrate surface chemistry was blocked by incubating the substrates for $10 \mathrm{~min}$ in Tris-buffered saline (TBS) (20 mM Tris-HCl, $\mathrm{pH} 7.5$, $150 \mathrm{mM} \mathrm{NaCl}$; Sigma-Aldrich) each supplemented with $0.1 \%(\mathrm{w} / \mathrm{v})$ bovine serum albumin (BSA) fraction $\mathrm{V}$ and $0.1 \%$ (v/v) Tween $^{\circledR} 20$ (both from Sigma-Aldrich), followed by a rinse in Milli-Q water. After spin drying, the 10 individual arrays were separated with hydrophobic borders (PAP PEN ${ }^{\circledR}$; The Binding Site, Birmingham, UK), unless otherwise indicated. The processed microarray substrates were stored in dry conditions until use.

In terms of $\mathrm{IC}_{50}$ value (i.e., the concentration of analyte for which the signal was decreased by $50 \%$ ), immunomicroarray sensitivity was determined with dilutions of pesticide standards. The standards consisted of Milli-Q water spiked with dilutions between $100 \mathrm{fg} / \mathrm{L}$ and $100 \mathrm{~g} / \mathrm{L}$ of the pesticide in question. For immunomicroarray quantification, $6 \mu \mathrm{L}$ of sample/standard were preincubated for 10 min with $6 \mu \mathrm{L}$ of Cy5 MAbs, diluted 25,000-fold (40 ng/mL) in TBS incubation buffer, supplemented with concentrations of $0.05 \%$ each (w/ v) BSA fraction $\mathrm{V}$ and (v/v) Tween 20, unless otherwise indicated, and then exposed to the arrays of the immobilized haptens for $60 \mathrm{~min}$. If secondary Cy3-labeled polyclonal anti-mouse antibodies were used for detection, then the arrays were incubated for 45 min at room temperature in a 10,000 fold $(100 \mathrm{ng} / \mathrm{mL})$ dilution of the secondary antibodies in TBS buffer and supplemented with $0.05 \%(\mathrm{w} / \mathrm{v}) \mathrm{BSA}$ fraction $\mathrm{V}$ and $0.05 \%(\mathrm{v} / \mathrm{v})$ Tween 20. After washing with TBS supplemented with $0.1 \%$ (w/v) Tween 20 for $10 \mathrm{~min}$, the substrates were rinsed with Milli$\mathrm{Q}$ water and spun dry.

Fluorescent MAb Cy5 and polyclonal $\mathrm{Cy} 3$ emissions were acquired with a ScanArray ${ }^{\circledR}$ Lite laser scanner (PerkinElmer Life Sciences, Boston, MA, USA) and quantified with Optiquant ${ }^{\circledR} 3.0$ (PerkinElmer Life Sciences). Twice the value of mean background fluorescence was subtracted from all acquired signals. The standard values were used to generate a semi-log graph based on the 4-parameter-logistic method by Rodbard (14) for determining the individual $\mathrm{IC}_{50}$ values. The standard curves and 4-parameter-logistic fits were generated with Origin ${ }^{\circledR} 6.1$ software (OriginLab, Northampton, MA, USA).

\section{RESULTS AND DISCUSSION}

The immunomicroarray was developed to replace a microplate-based immunoassay. To obtain optimum competitive immunomicroarray conditions, the impact of several assay parameters, 
such as F/A ratio, sample incubation buffer, spotting buffer, and microarray substrate properties, were studied. The influences of antibody concentration, incubation time, and temperature on immunomicroarray sensitivity and precision were also investigated during immunomicroarray development (E. Belleville, M. Dufva, J. Aamand, L. Bruun, L. Clausen, and C. Christensen, unpublished data).

\section{Array Incubation Format}

We evaluated several different array incubation formats [i.e., conventional glass coverslips, GeneFrames (Abgene, Surrey, UK), and uncovered hydrophobic barriers]. The highest signals as well as lowest variance were obtained with uncovered arrays simply separated by hydrophobic barriers, which were made with a hydrophobic pen. This format was the least time-consuming and least expensive technique. Furthermore, it offered the optional highest density of individual arrays per slide (data not shown).

\section{Fluorescent Monoclonal Antibodies}

Fluorescent labels such as cyanine dyes have proven their high sensitivity in DNA and protein chip applications $(15,16)$. We adopted fluorescent labels for the immunomicroarray and thereby substituted the secondary ELISA signal generation step by directly labeling the primary MAbs with Cy5 dye.

Two types of Cy5 labeling kits were compared. One attaches Cy5 labels to free amino groups and the other, which is specifically designed for labeling MAbs, attaches Cy5 maleimide dyes to free sulfhydryl (SH) groups. Two batches of the same anti-BAM MAb clone were labeled with both dye kits, and the resulting F/A ratios were spectrometrically determined. The different monoreactive dyes yielded significant differences in F/A ratios. The first resulted in an F/A ratio of 6.5 compared to the F/A ratio of 1.5 that was observed for the second kit. The two differently labeled antibodies were exposed to dilutions of BAM standards to evaluate how different labeling methods affected the signal intensity and $\mathrm{IC}_{50}$ value. The antibody with the lower F/A ratio produced 3-fold lower $\mathrm{IC}_{50}$ values and a 2 -fold increase in maximum fluorescence (Figure 1). The immunomicroarray results confirmed previous results in which F/A ratios between 1 and 2 provided the highest signals, while higher ratios appeared to quench fluorescence and/or affect MAb affinity (17). The difference between labeling techniques in terms of F/A ratios and their corresponding effects on immunomicroarray sensitivity might also be attributed to the difference in dye binding chemistry. The higher F/A ratio was obtained from dyes binding to free amino groups, which are also integral parts of the antigen binding groove of the Fab fragment; therefore, MAb affinity might be affected. The lower F/A ratio resulted from the specific dye attachment to free SH groups. Amino acids with SH groups are substantially less frequent and are usually not part of the antibody recognition pocket.

Most immunoassays performed in microplates or on microarrays are

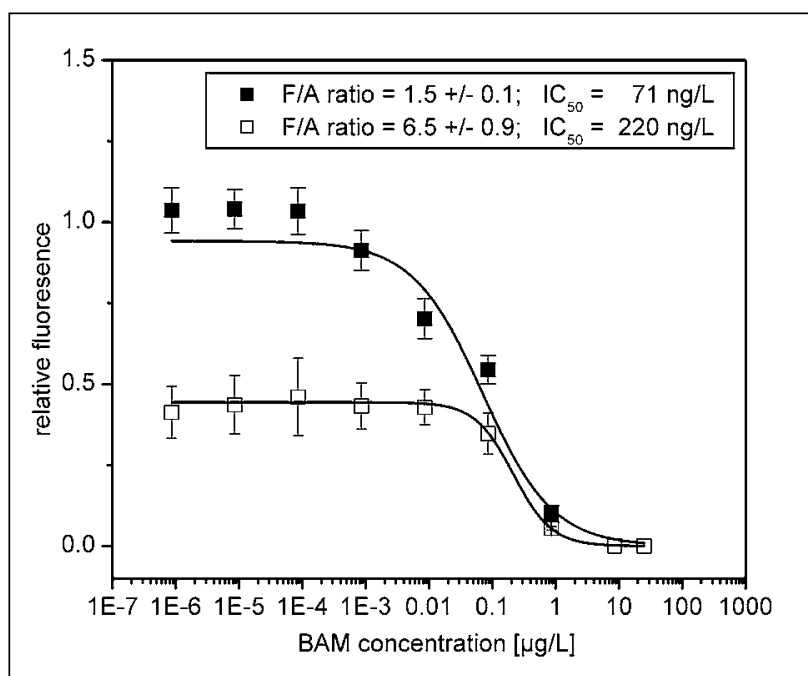

Figure 1. Monoclonal antibody (MAb) Cy5 labeling ratios. Anti2,6-dichlorobenzamide (BAM) MAbs from the same clone were labeled with two types of Cy5 monoreactive dye kits. After purification, fluorochrome/antibody (F/A) labeling ratios were determined spectrophotometrically. Both MAb batches were tested by competitive BAM immunomicroarray by exposing them to a dilution series of BAM. Fitted standard curves are shown. Immunomicroarray sensitivities, defined as the $\mathrm{IC}_{50}$ value (i.e., the concentration of analyte for which the signal was decreased by $50 \%$ ), were derived from the individual standard curves. The black squares represent the results for a $1.5 \mathrm{~F} / \mathrm{A}$ ratio, while the white squares represent the results for a $6.5 \mathrm{~F} / \mathrm{A}$ ratio. The results are based on four separate dye labeling experiments, each experiment analyzed on four slides that included six replicate spots per concentration. The error bars represent the mean $(\bar{x} \pm S D)$.

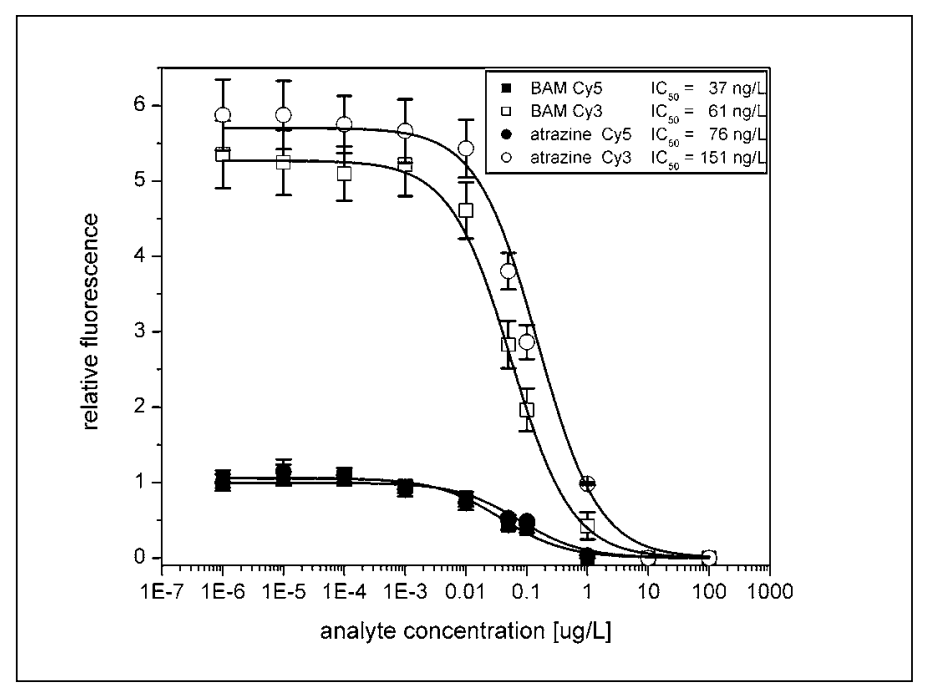

Figure 2. Primary and secondary signal quantification. Both the 2,6-dichlorobenzamide (BAM) and atrazine immunomicroarrays were used to compare $\mathrm{IC}_{50}$ values (i.e., the concentration of analyte for which the signal was decreased by $50 \%$ ) obtained from directly Cy5-labeled primary monoclonal antibodies (MAbs) in comparison to $\mathrm{IC}_{50}$ values obtained with a secondary Cy3-labeled goat-anti-mouse polyclonal antibody. First, primary Cy5-labeled anti-BAM and anti-atrazine MAbs were exposed to a dilution series of either BAM or atrazine. Following incubation, fluorescent Cy5 signals were obtained. Afterwards, the same arrays were incubated with the secondary $\mathrm{Cy} 3$ polyclonal goat-anti-mouse antibody, and the resulting $\mathrm{Cy} 3$ fluorescence was obtained. Fitted standard curves are shown. The immunomicroarray $\mathrm{IC}_{50}$ values were derived from the individual standard curves. The results are based on five experiments on EasySpot microarray substrates that included six replicate spots per concentration. The error bars represent the mean $(\bar{x} \pm S D)$. 


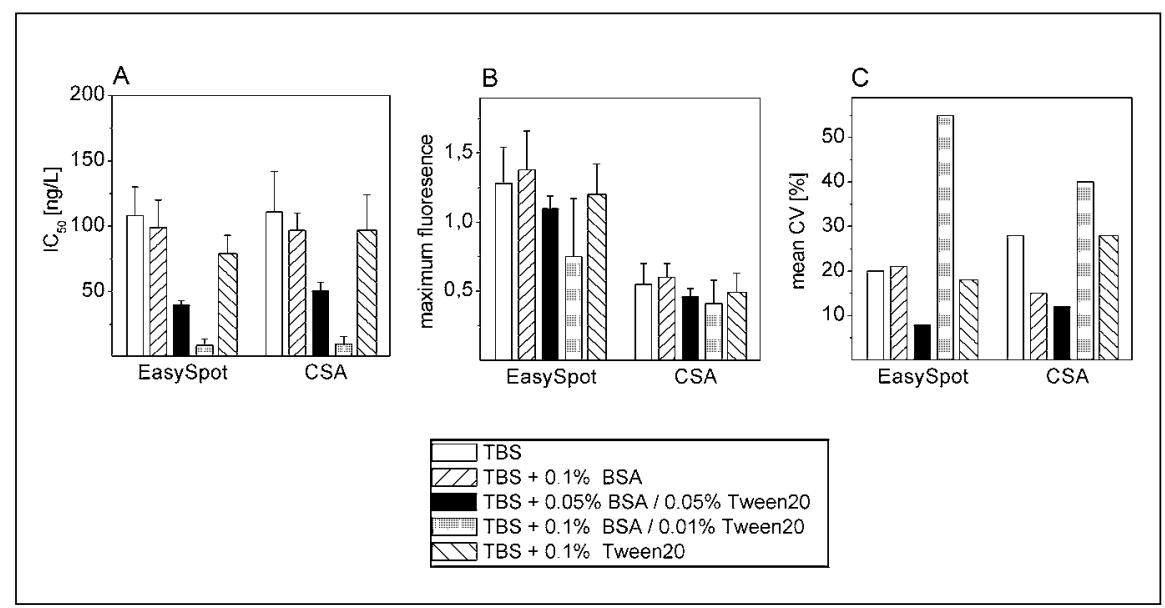

Figure 3. Sample incubation buffer comparison. The competitive 2,6-dichlorobenzamide (BAM) immunomicroarray was used to assess the effect of buffer additives on $\mathrm{IC}_{50}$ (i.e., the concentration of analyte for which the signal was decreased by $50 \%$ ), maximum signal, and variance. The incubation buffers compared were identical in their basic tris-buffered saline (TBS) composition, and only the concentrations of the bovine serum albumin (BSA) and Tween 20 additives varied as indicated. (A) $\mathrm{IC}_{50}$ values, (B) maximum fluorescence, and (C) variance are shown in relation to the sample buffer composition. The results are based on 10 EasySpot and CSA microarray substrates that included 6 replicate spots per concentration. The error bars represent the mean $(\bar{x} \pm S D)$.

based on either secondary enzymatically generated signals or on the binding of secondary labeled polyclonal antibodies. In the case of the BAM immunomicroarray, $\mathrm{IC}_{50}$ values of $37 \mathrm{ng} / \mathrm{L}$ (Figure 2) were 5-fold lower compared to its microplate-based ELISA version (13).

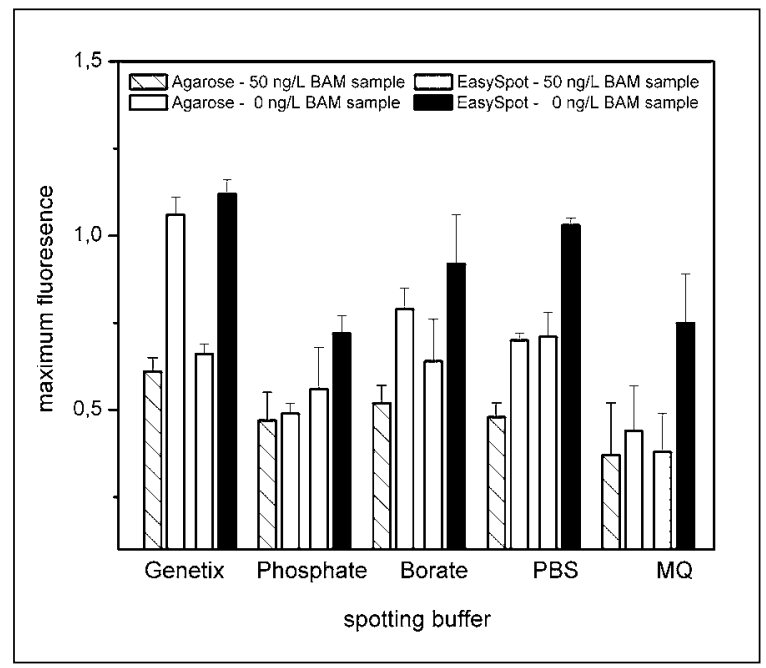

Figure 4. Spotting buffer comparison. Five different spotting buffers were compared for their impact on the 2,6-dichlorobenzamide (BAM) immunomicroarray performance. The competitive BAM immunomicroarray was used to compare quantification values for two BAM samples of different concentrations. The acquired fluorescence values of the individual samples are shown in relation to the spotting buffer and substrate type. The results are based on 3 experiments using EasySpot and activated agarose microarray substrates; each concentration was tested in 24 replicates per experiment. The error bars represent the mean $(\bar{x} \pm$ SD). PBS, phosphatebuffered saline; MQ, Milli-Q water.
To clarify whether this gain in sensitivity observed for the immunomicroarray can be explained by the use of directly labeled MAbs, we compared $\mathrm{IC}_{50}$ valacquired from primary $\mathrm{Cy} 5$-labeled mouse-anti-BAM MAbs to secondary Cy3-labeled anti-mouse polyclonal antibodies. To identify whether results purely correlated to one primary antibody-antigen pair, we also performed the identical experiment for an atrazine immunomicroarray. Results were identical for both pesticides (Figure 2). Immunomicroarray curves obtained using Cy3-labeled secondary antibodies led up to a 2.5 -fold increase in $\mathrm{IC}_{50}$ values and nearly 6-fold higher maximum fluorescence compared to the values obtained with directly Cy5 labeled primary MAbs. In another experimental series, we substituted the primary Cy5-labeled antibodies with corresponding unlabeled antibodies of the same clones and performed the same secondary incubation (data not shown). Whether the 
primary labeled MAb was labeled or not did not have an effect on the binding of the secondary antibody, secondary binding affinity was apparently not altered by existing primary Cy5 labels. Although it is easier and less expensive to label one secondary polyclonal antibody in contrast to labeling all primary specific MAbs individually, the results indicated that the direct labeling of the primary MAb increased sensitivity and simplified and accelerated the assay. The results also showed that the use of directly labeled MAbs could partly explain the higher sensitivity of the immunomicroarray compared to the ELISA.
While the ambient analyte theory (4) applies primarily to noncompetitive assays, it also supports the improvement in sensitivity for the competitive immunomicroarray.

\section{Sample Buffer Composition}

The next step in immunomicroarray characterization was the sample buffer composition. Tween 20, a nonionic surfactant, and BSA are the two most commonly used buffer additives in immunoassays for reducing nonspecific interactions. Nonoptimal concentrations of both additives in ELISAs re- duced maximum signals and increased the $\mathrm{IC}_{50}$ value; thus, previous literature recommended excluding them or to specifically identify the optimum concentrations (18-20).

To evaluate any influence of BSA and Tween 20 on immunomicroarray performance, the microarrays were incubated with a dilution series of BAM in different TBS buffers (Figure 3). The results showed that the addition of $0.05 \%$ each BSA and Tween 20 gave an intermediate $\mathrm{IC}_{50}$ value (Figure $3 \mathrm{~A}$ ) but very low variance (Figure $3 \mathrm{C}$ ). The buffer composition, however, did not influence the individual signal intensities

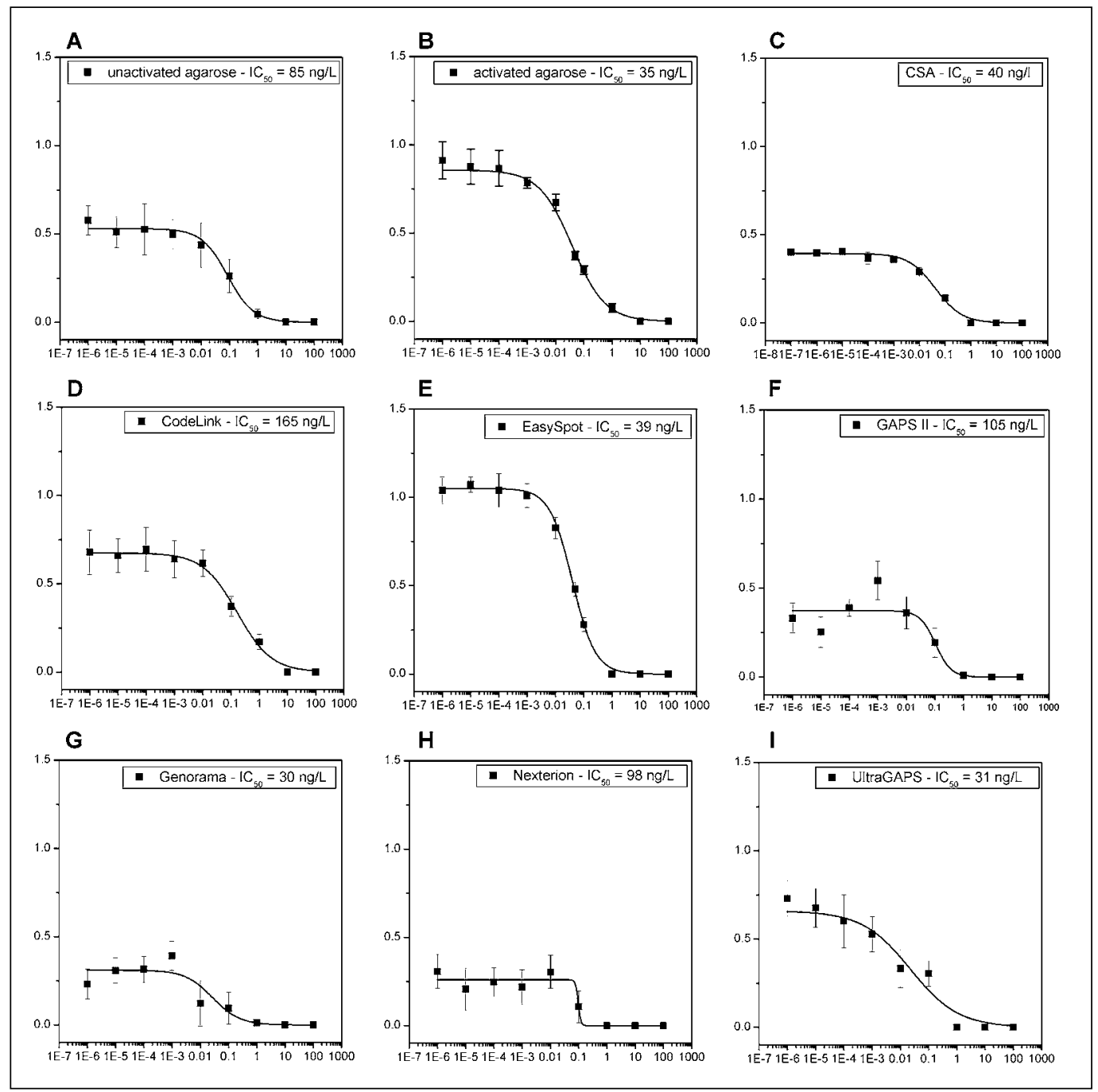

Figure 5. Microarray substrate comparison. The 2,6-dichlorobenzamide (BAM) immunomicroarray was used to compare the impact of nine microarray substrates of various surface chemistries (see Table 1) on immunomicroarray performance. The microarray substrates were tested with the competitive BAM immunomicroarray by exposing them to a dilution series of BAM. Corresponding fitted standard curves as well as $\mathrm{IC}_{50}$ values (i.e., the concentration of analyte for which the signal was decreased by 50\%) are shown for every substrate type. The results are based on 10 experiments on individual substrates that included 6 replicate spots per concentration. The $\mathrm{x}$-axes represent the individual standard concentrations in micrograms per liter, while the $y$-axes represent the corresponding fluorescence signals. The error bars represent the mean $(\overline{\times} \pm \mathrm{SD})$.
(Figure 3B). Although the addition of each $0.1 \%$ BSA and Tween 20 to the sample buffer resulted in the lowest $\mathrm{IC}_{50}$ value (Figure 3A), the variance was up to $55 \%$ and, thus, unacceptable (Figure 3C). Furthermore, the impact of sample buffer composition on the immunomicroarray performance was identical on both EasySpot Universal ${ }^{\circledR}$ (U-Vision Biotech, Taipei, Taiwan) and CSA $^{\circledR}$ (TeleChem International) substrates, which suggests that the effect of the incubation buffer composition was independent of substrate surface chemistry (Figure 3 ). The only difference was the reduced fluorescent signals on CSA substrates (Figure 3B).

\section{Spotting Buffer}

The spotting buffer can influence aspects of array quality, such as the protein-binding capacity of a surface, the stability of printed proteins, and the spot morphology $(21,22)$. We tested five spotting buffers, the amine spotting solution, $300 \mathrm{mM}$ phosphate, $150 \mathrm{mM}$ each borate, PBS, and Milli-Q water, on both activated 


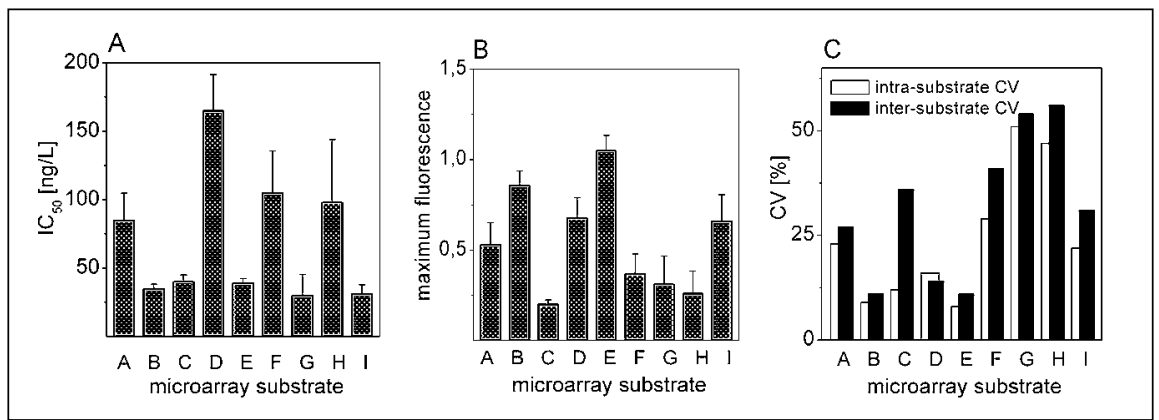

Figure 6. $\mathrm{IC}_{50}$, maximum fluorescence, and coefficient of variation (cv) in relation to microarray substrate. The figures comprise the evaluation of the results given in Figure 5. (A) $\mathrm{IC}_{50}$ values, (B) maximum fluorescence, as well as (C) intra-substrate and inter-substrate variance are shown in relation to microarray substrate type. Error bars represent the mean standard deviation.

agarose aldehyde substrates and on EasySpot-activated epoxy silane surfaces. Immunomicroarray maximum fluorescence was compared for different BAM sample concentrations (i.e., $50 \mathrm{ng} / \mathrm{L}$ representing a concentration close to the actual immunomicroarray $\mathrm{IC}_{50}$ value) ranging between 35 and 39 (Figure 5, B and E) and $0 \mathrm{ng} / \mathrm{L}$, which provided maximum competitive assay fluorescence. Thus, a fluorescence ratio of around 2 between the signal obtained from the $0 \mathrm{ng} / \mathrm{L}$ sample and the signal obtained from the $50 \mathrm{ng} / \mathrm{L}$ sample is expected. Figure 4 shows the results obtained for both BAM samples in relation to the spotting buffer. Signal intensity, fluorescence ratios, and variance were nearly independent of substrate type. Using the Genetix Amine Spotting Solution or the commonly used PBS buffer $(9,22)$ resulted in the highest signals as well as the lowest variance. Spots made using these two buffers also resulted in the highest fluorescent ratios.

\section{Microarray Substrate Comparison}

To carry out reproducible and reliable immunoassays on a microarray, it is necessary to immobilize antibodies, proteins, or other antigens in a way that results in efficient deposition without jeopardizing the recognition or receptor functionality. The printing of antibody/antigen microarrays has been reported on a variety of surfaces and immobilization chemistries. Twodimensional (2-D) surfaces with amino $(22,23)$, aldehyde- (3), or polylysine$(11,24)$ cross-linkers have shown good reproducibility, efficient immobilization, and low background when used in conjunction with fluorescence detection. Alternatively, several threedimensional (3-D) microarray surfaces have been described, based on their established performance in traditional biochemical analyses. Polyvinylidene difluoride (PVDF) and nitrocellulose have shown to be incompatible for protein microarrays $(10,23,25,26)$. Suitable high-protein densities could not be established, spotted material spread across the surface, and/or unacceptable high background was observed $(23,27,28)$. However, agarose and acrylamide were described to provide highly porous and hydrophilic 3-D surface matrices that are suitable for DNA and protein microarrays $(24,29,30)$.

Several commercially available microarray substrates with various surface chemistries were tested for the identification of the best immunomicroarray substrate (Table 1). All surfaces were tested identically by comparing their BAM immunomicroarray performance with different dilutions of BAM standards. Figure 5 shows immunomicroarray standard curves, and the summary of key parameters, such as $\mathrm{IC}_{50}$ values, maximum fluorescence, and variance is shown in Figure 6. Only two of the compared surfaces fulfilled the required immunomicroarray criteria of a low $\mathrm{IC}_{50}$ value, low variance, and strong signals. EasySpot substrates (E) and the inexpensive, activated agarose substrates (B) resulted in the lowest $\mathrm{IC}_{50}$ values (Figure 6A), lowest variance (Figure $6 \mathrm{C}$ ) and, at the same time, the strongest signals (Figure 6B). Comparably low $\mathrm{IC}_{50}$ values were also obtained on CSA substrates (C) in spite of relatively low fluorescence values. However, a significant inter-batch variance suggested that this substrate was less suitable. Of the remaining substrate types, Genorama SAL- ${ }^{\circledR}$ (Asper Biotech, Tartu, Estonia) (G) and UltraGAPS $^{\circledR}$ (I) (Corning, Corning, NY, USA) also achieved low $\mathrm{IC}_{50}$ values; however, these substrates showed high variances and, thus, excluded their immunomicroarray suitability. A clear correlation between surface chemistry and array performance was not apparent because the optimal immunomicroarray results were obtained on both agarose aldehyde surfaces and EasySpot epoxy silane surfaces. However, the various types of amino silane linkers as well as the hydroxyl surface of the inactivated agarose slide were obviously unsuitable for the immunomicroarray.

Consistent with our results, aldehyde (31) and epoxy surfaces have been shown to provide efficient binding chemistries. However, glycidoxypropyl (22), amino silane, aldehyde, and polylysine surfaces have been described as performing well (24).

In summary, immunomicroarray results showed that assay performance can vary significantly even for closely related surface chemistries. In-house fabricated agarose substrates performed equally well to commercially available substrates; however, agarose substrates were inexpensive in comparison.

\section{ACKNOWLEDGMENTS}

This work was financially supported by the Danish Research Council (SUE project no. 2027-00-0005 and DABIC project no. 2014-00-0003).

\section{REFERENCES}

1.Ramsay, G. 1998. DNA chips: state-of-the art. Nat. Biotechnol. 16:40-44.

2.Chen, G.Y.J., M. Uttamchandani, R.Y. Lue, M.L. Lesaicherrea, and S.Q. Yao. 2003. Array-based technologies and their applications in proteomics. Curr. Top. Med. Chem. 3:705-724.

3.MacBeath, G. and S.L. Schreiber. 2000. Printing proteins as microarrays for highthroughput function determination. Science 289:1760-1763.

4.Ekins, R., F. Chu, and E. Biggart. 1990. Multispot, multianalyte, immunoassay. Ann. Biol. Clin. (Paris) 48:655-666.

5.Mendoza, L.G., P. McQuary, A. Mongan, R. 
Gangadharan, S. Brignac, and M. Eggers. 1999. High-throughput microarray-based enzyme-linked immunosorbent assay (ELISA). BioTechniques 27:778-788.

6.Mullenix, M.C., S. Wiltshire, W.P. Shao, G. Kitos, and B. Schweitzer. 2001. Allergenspecific $\operatorname{IgE}$ detection on microarrays using rolling circle amplification: correlation with in vitro assays for serum IgE. Clin. Chem. 47: 1926-1929.

7.Silzel, J.W., B. Cercek, C. Dodson, T. Tsay, and R.J. Obremski. 1998. Mass-sensing, multianalyte microarray immunoassay with imaging detection. Clin. Chem. 44:2036-2043.

8.de Wildt, R.M., C.R. Mundy, B.D. Gorick, and I.M. Tomlinson. 2000. Antibody arrays for high-throughput screening of antibodyantigen interactions. Nat. Biotechnol. 18: 989-994.

9.Joos, T.O., M. Schrenk, P. Hopfl, K. Kroger, U. Chowdhury, D. Stoll, D. Schorner, M. Durr, et al. 2000. A microarray enzyme-linked immunosorbent assay for autoimmune diagnostics. Electrophoresis 21:2641-2650.

10.Chen, G.Y.J., M. Uttamchandani, Q. Zhu, G. Wang, and S.Q. Yao. 2003. Developing a strategy for activity-based detection of enzymes in a protein microarray. Chem. Biochem. 4:336-339.

11.Haab, B.B., M.J. Dunham, and P.O. Brown. 2001. Protein microarrays for highly parallel detection and quantitation of specific proteins and antibodies in complex solutions. Genome Biol. 2:1-13.

12.Bruun, L., C. Koch, M.H. Jakobsen, B. Pedersen, M. Christiansen, and J. Aamand. 2001. Characterization of monoclonal antibodies raised against different structures belonging to the s-triazine group of herbicides. Anal. Chim. Acta 436:87-101

13.Bruun, L., C. Koch, B. Pedersen, M.H. Jakobsen, and J. Aamand. 2000. A quantitative enzyme-linked immunoassay for the detection of 2,6-dichlorobenzamide (BAM), a degradation product of the herbicide dichlobenil. J. Immunol. Methods 240:133-142.

14.Rodbard, D. 1974. Statistical quality-control and routine data-processing for radioimmunoassays and immunoradiometric assays. Clin. Chem. 20:1255-1270.

15.Lam, K.S. and M. Renil. 2002. From combinatorial chemistry to chemical microarray. Curr. Opin. Chem. Biol. 6:353-358.

16.MacBeath, G. 2002. Protein microarrays and proteomics. Nat. Genet. 32(Suppl):526-532.

17.Gruber, H.J., C.D. Hahn, G. Kada, C.K. Riener, G.S. Harms, W. Ahrer, T.G. Dax, and H.G. Knaus. 2000. Anomalous fluorescence enhancement of Cy 3 and Cy 3.5 versus anomalous fluorescence loss of Cy5 and Cy7 upon covalent linking to IgG and noncovalent binding to avidin. Bioconjug. Chem. 11:696-704.

18.Lee, N., D.P. McAdam, and J.H. Skerritt. 1998. Development of immunoassays for type II synthetic pyrethroids. 1. Hapten fesign and spplication to heterologous and homologous assays. J. Agric. Food Chem. 46:520-534.

19.Mercader, J.V. and A. Montoya. 1997. A monoclonal antibody-based ELISA for the analysis of azinphos-methyl in fruit juices. Anal. Chim. Acta 347:95-101.

20.Mercader, J.V. and A. Montoya. 1999.
Development of monoclonal ELISAs for azinphos-methyl. 2. Assay optimization and water sample analysis. J. Agric. Food Chem. 47:1285-1293.

21.Delehanty, J.B. and F.S. Ligler. 2003. Method for printing functional protein microarrays. BioTechniques 34:380-385.

22.Kusnezow, W., A. Jacob, A. Walijew, F. Diehl, and J.D. Hoheisel. 2003. Antibody microarrays: an evaluation of production parameters. Proteomics 3:254-264.

23.Schweitzer, B. and S.F. Kingsmore. 2002. Measuring proteins on microarrays. Curr. Opin. Biotechnol. 13:14-19.

24.Angenendt, P., J. Glokler, D. Murphy, H. Lehrach, and D.J. Cahill. 2002. Toward optimized antibody microarrays: a comparison of current microarray support materials. Anal. Biochem. 309:253-260.

25.Ge, H. 2000. UPA, a universal protein array system for quantitative detection of proteinprotein, protein-DNA, protein-RNA and protein-ligand interactions. Nucleic Acids Res. 28:E3.

26.Mercader, J.V. and A. Montoya. 1999. Development of monoclonal ELISAs for azinphosmethyl. 1. Hapten synthesis and antibody production. J. Agric. Food Chem. 47:1276-1284.

27.Lueking, A., M. Horn, H. Eickhoff, K. Bussow, H. Lehrach, and G. Walter. 1999. Protein microarrays for gene expression and antibody screening. Anal. Biochem. 270: 103-111.

28.Templin, M.F., D. Stoll, M. Schrenk, P.C. Traub, C.F. Vohringer, and T.O. Joos. 2002. Protein microarray technology. Drug Discov. Today 7:815-822.

29.Afanassiev, V., V. Hanemann, and S. Wolfl. 2000. Preparation of DNA and protein micro arrays on glass slides coated with an agarose film. Nucleic Acids Res. 28:E66.

30.Zhu, H. and M. Snyder. 2003. Protein chip technology. Curr. Opin. Chem. Biol. 7:55-63.

31.MacBeath, G., A.N. Koehler, and S.L. Schreiber. 1999. Printing small molecules as microarrays and detecting protein-ligand interactions en masse. J. Am. Chem. Soc. 121: 7967-7968.

Received 21 May 2003; accepted 9 September 2003.

\section{Address correspondence to:}

Claus B.V. Christensen

BioArray Project

Mikroelektronik Centret

Ørsteds Plads 345 East

DK-2800 Kgs. Lyngby, Denmark

e-mail: cbc@mic.dtu.dk 